\title{
An Effective Channel Allocation Scheme to Reduce Co-Channel and Adjacent Channel Interference for WMN Backhaul
}

\author{
SHAZIA ABBASI*, IMDAD ALI ISMAILI*, AND FAHIM YAR KHUHAWAR** \\ RECEIVED ON 16.04.2015 ACCEPTED ON 14.12.2015 \\ ABSTRACT
}

\begin{abstract}
Two folded work presents channel allocation scheme sustaining channel orthogonality and channel spacing to reduce CCI (Co-Channel Interference) and ACI (Adjacent Channel Interference) for inter flow of an intra-flow link.

Proposed scheme as a part of radio resource allocation is applied on infrastructure based backhaul of wireless mesh network using directional antennas. The proposed approach is applied separately on 2.4 and $5 \mathrm{GHz}$ bands. Interference of connectivity graph is modelled by strongly connected directed graph and greedy algorithms are used for channel allocation. We have used OPNET Modeller suite to simulate network models for this research. The proposed arrangement reduces the channel interference and increases system throughput. In this research, the influence of channel is computed in terms of network throughput and delay.
\end{abstract}

Key Words: Adjacent Channel Interference, Backhaul, Co-Channel Interference, Multi-Radio, Throughput, Wireless Mesh Networks.

\section{INTRODUCTION}

T The wireless network is designed to recreate the high superiority communications that is characteristically delivered by wired networks [12]. High quality communications are identified with continuous network connections, reliable delivery of data and high throughput in that view MRMC (Multi Radio Multi Channel) wireless Network offers such quality, however; interference has become one of the main sources to degrade the network performance [3]. In these networks, the interference can be controlled by two main functions: power limitations and channel allocation [4]. This paper only considers channel allocation to reduce interference in MRMC. Channel allocation in MRMC network deals to assign different channels to interfering links [5], which reduces the interference among parallel transmissions. In this paper, a framework for backhaul of MRMC mesh network is proposed to minimize interference by two levels. Fig. 1 shows the steps to handle backhaul interference; at physical level, interference area is confined with directional antennas and then further controlled at logical level by channel allocation. In the channel allocation, partially overlapped channels are assigned to links which maintain orthogonality to reduce CCI while channels are appropriately spaced to control ACI.

* Institute of Information \& Communication Technology, University of Sindh, Jamshoro.

** Deparment of Telecommunication Engineering, Mehran University of Engineering \& Technology, Jamshoro.

Mehran University Research Journal of Engineering \& Technology, Volume 35, No. 4, October, 2016 [p-ISSN: 0254-7821, e-ISSN: 2413-7219] 
Proposed work is deeming a way to reduce CCI and ACI. Fundamentally, co-channel interference is controlled by maintaining orthogonality of channels and ACI is controlled by proper channel spacing assigned to nodes. In our work we consider both interferences and assign channels to nodes with an attention of orthogonality and channel space. The suggested approach is applied on two ISM (Industrial, Scientific and Medical) bands; 2.4 and $5 \mathrm{GHz}$. When it was applied on $2.4 \mathrm{GHz}$ band it focused to orthogonality of channels as this band offers only 3 orthogonal channels. However, when approach was applied on $5 \mathrm{GHz}$ band it focuses to channel spacing because all available channels of band are orthogonal in nature so there is need to assign the channels with proper channel spacing.

The rest of the paper consists of six sections; Section 2 describes literature review. Proposed frame work for channel assignment is presented in section 3; this section further comprises the formation of connectivity graph, interference nodes and conflict graph. System design is introduced in section 4 and simulation results and discussion are presented in section 5. Finally conclusion is drawn in section 6 .

\section{RESEARCH REVIEW}

Several approaches are developed to address the issue of interference in MRMC wireless networks. Many

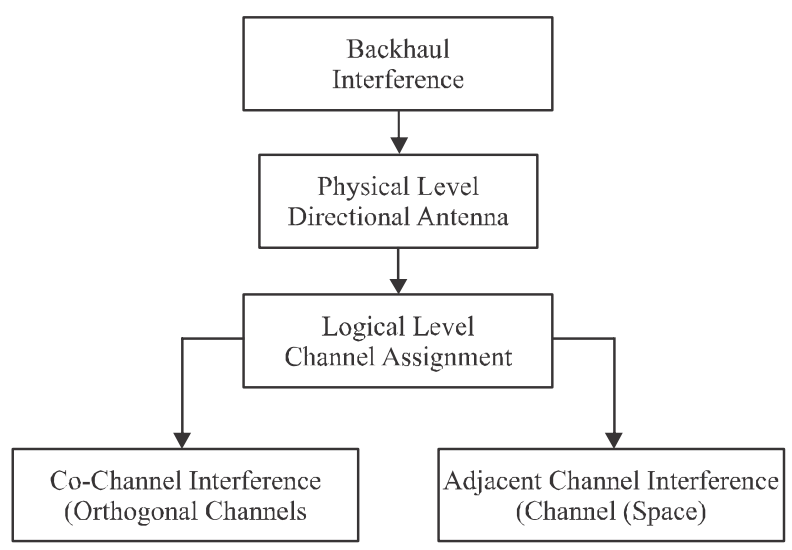

FIG. 1. PROPOSED SCHEME authors such as from [6-10] reported that systems offer more capacity when they use partially overlapped channels with the support of non-overlapped. Authors propose a channel assignment technique which is framed jointly with network coding and broadcast link scheduling [6]. The aim of work was to enhance throughput.

A joint formulation of channel assignment and end to end flow allocation is presented in [7], this work shows that mixed use of overlapping and non-overlapping channels enhance the system throughput. The formulation is based on number of parameters such as transmission power, Signal t interference and noise ratio, path loss component and filter response of nodes. A heuristic based channel assignment is presented in [8], the work explores the way to use of partially overlapped channels in a way to increase system capacity, they present an interference model, termed as I-Matrix and propose a channel assignment for that. Focused work of [9] uses SIN (Signal to Interference and Noise) model for channel assignment of partially overlapped channels. Their effort increases throughput with the control of total interference. However, in [10], authors use game theory to solve channel assignment problem, they proposed distributed algorithm for the assignment of partially overlapped channels.

Literature from [11-16] discussed some algorithms for channel assignment with the consideration of control of interference. A distributed algorithm for channel assignment is proposed in [11], they use game theory for channel assignment, the algorithm uses confined information of load to get close to ideal performance. In the work of [12], an efficient management architecture is presented and suggested a routing method for WMNs which project to load balancing of traffic. The authors attempted to maximize throughput with careful balancing of network traffic. A centralized CA and routing algorithm is presented in [13]. The authors proposed an ILP (Integer Linear Programming) model which was not related to traffic demands. The target of work was to 
maximize the throughput of network. The work of [14] suggested a channel allocation method which is based on radio frequency slotting. Work oriented to reduce CCI by slotting received and transmitted packets are split by two channels. Efficient utilization of resource is focused in [15] where node power and its assigned channel are used as factors to enhance the overall throughput of networks in terms of traffic flows. Research presented in [16] concentrated to formulate another combination for join work. They combined the channel assignment with flow distribution and focus of research was to enhance throughput and reducing queuing delay.

\section{PROPOSED FRAMEWORK}

The proposed work attempt to present a frame work for channel allocation to reduce interference among the backhaul links: Interference is controlled by directional antennas at fundamental level, than scheme for channel allocation is proposed to reduce further interference introduced by intra-flow and inter-flow links. In the proposed framework every backhaul node connected with 2 bidirectional antenna, each node has two meaning of interference: interference of direct nodes and the nodes placed in the access direction of antenna.

\subsection{Connectivity Graph}

The WMN nodes with multiple interfaces are taking as vertices set V, (Two for proposed work), these nodes are connected with links known as edges and represented as set E.

Communication between nodes $\mathrm{v}_{\mathrm{i}}$, and $\mathrm{v}_{\mathrm{j}}$ is only possible by link e under three conditions:

(1) When $v_{i}$, and $v_{j}$ are member vertices set $V$

$\left(\mathrm{v}_{\mathrm{i},}, \mathrm{v}_{\mathrm{j}}\right) " \mathrm{~V}$

(2) If both nodes are in transmission range $r_{\text {ic }}$.

$\mathrm{v}_{\mathrm{i}}-\mathrm{v}_{\mathrm{j}}<\mathrm{r}_{\mathrm{ic}}$
(3) If both employing identical channels.

$\mathrm{v}_{\mathrm{i}(\mathrm{ic})}=\mathrm{v}_{\mathrm{j} \text { (ic) }}$

(where $\mathrm{i}_{\mathrm{c}}$ shows identical channels)

Connectivity graph $\mathrm{G}$ of Fig. 2 contain nodes from A-H

\subsection{Interference Nodes}

For proper channel allocation the connectivity graph is modelled to identify the links in conflict which are sources to generate interference. For the proposed work the interference range for every node is up to $2^{\text {nd }}$ hop of inter flow; IFu and IF(u+1) and single hop of intra flow IFv.

For such condition the interference at node is identified as:

$\mathrm{IF}($ node $)=\mathrm{u}, \mathrm{u}+1, \mathrm{v}$

Where $\mathrm{u}, \mathrm{u}+1$ and $\mathrm{v}$ " $\mathrm{V}$.

Backhaul is connected with directional antennas so that interference links considered with flow direction of nodes:

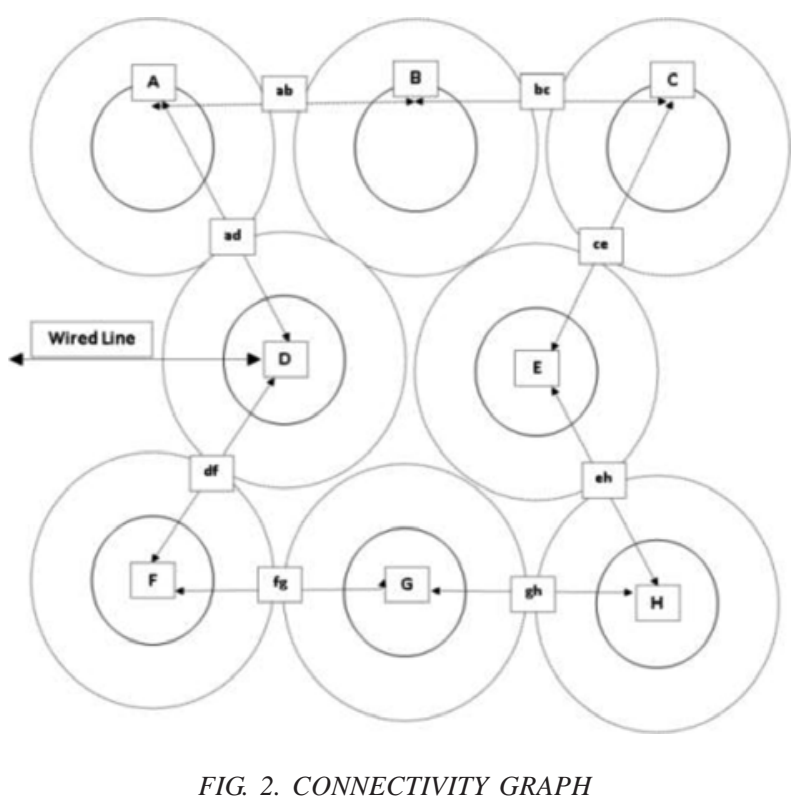

Mehran University Research Journal of Engineering \& Technology, Volume 35, No. 4, October, 2016 [p-ISSN: 0254-7821, e-ISSN: 2413-7219] 
$\mathrm{IF}(\mathrm{A})=\mathrm{ab}, \mathrm{bc}, \mathrm{ad}$

$\mathrm{IF}(\mathrm{B})=\mathrm{bc}, \mathrm{ab}$

$\mathrm{IF}(\mathrm{C})=\mathrm{ab}, \mathrm{bc}, \mathrm{ce}$

IF(D)ad, df

$\mathrm{IF}(\mathrm{E})=\mathrm{eh}, \mathrm{ce}$

$\mathrm{IF}(\mathrm{F})=\mathrm{df}, \mathrm{fg}, \mathrm{gh}$

$\mathrm{IF}(\mathrm{G})=\mathrm{fg}, \mathrm{gh}$

$\mathrm{IF}(\mathrm{H})=\mathrm{eh}, \mathrm{gh}$

\subsection{Conflict Graph}

Conflict graph is used to model interference of the network. Conflict graph $G_{c}$ is formed from connectivity graph $G$ highlighting the possible nodes and links in conflict with the consideration of interferences nodes. Conflict graph contains set of conflict vertices $\mathrm{V}_{\mathrm{c}}$ and set of conflict edges.

A node is said to be in conflict if their associated edges/ links are member of $\operatorname{In}(\mathrm{E})$. The nodes are said to be in conflict if their associated edges are working on same channel within interference area.

In Fig. 3, the conflict graph of conflict nodes and vertices are identified with the flow of traffic. Identified conflict nodes and vertices are shown in Equations (5-8).

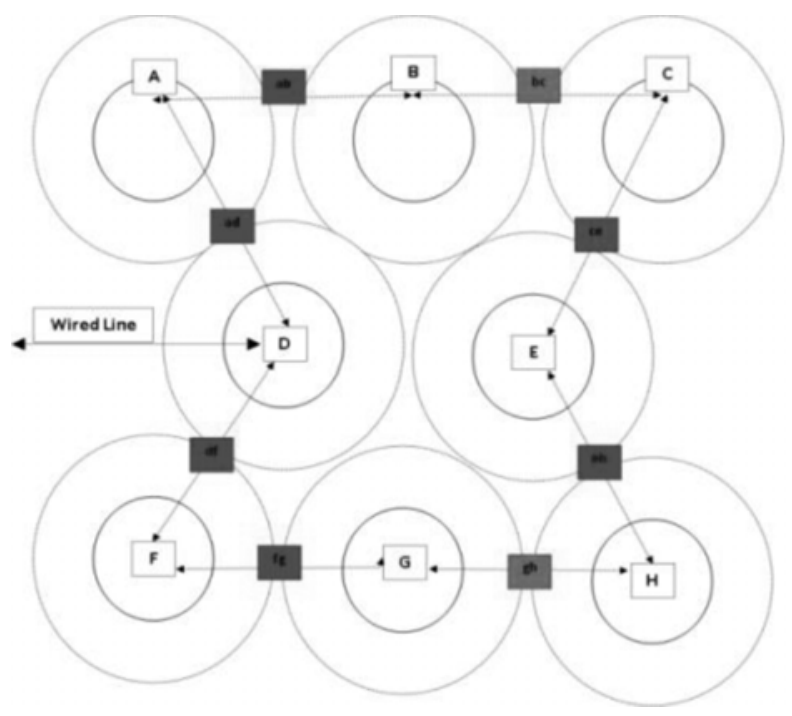

FIG. 3. CONFLICT GRAPH
Conflict nodes in clockwise direction are:

$\mathrm{Vc}($ clock $)=\mathrm{A} \rightarrow \mathrm{B} \rightarrow \mathrm{C} \rightarrow \mathrm{E} \rightarrow \mathrm{H} \rightarrow \mathrm{G} \rightarrow \mathrm{F} \rightarrow \mathrm{D}$

Conflict Nodes in anti-clockwise direction are:

$\mathrm{Vc}($ anti-clock $)=\mathrm{D} \rightarrow \mathrm{F} \rightarrow \mathrm{G} \rightarrow \mathrm{H} \rightarrow \mathrm{E} \rightarrow \mathrm{C} \rightarrow \mathrm{B} \rightarrow \mathrm{A}$

Conflict vertices in clockwise direction are:

$\mathrm{Ec}($ clock $)=\mathrm{ab} \rightarrow \mathrm{bc} \rightarrow \mathrm{ce} \rightarrow \mathrm{eh} \rightarrow \mathrm{gh} \rightarrow \mathrm{fg} \rightarrow \mathrm{df} \rightarrow \mathrm{ad}$

Conflict vertices in anti-clockwise direction are:

Ec(anti-clock $)=\mathrm{ad} \rightarrow \mathrm{df} \rightarrow \mathrm{fg} \rightarrow \mathrm{gh} \rightarrow \mathrm{eh} \rightarrow \mathrm{ce} \rightarrow \mathrm{bc} \rightarrow \mathrm{ab}$

\section{SYSTEM DESIGN}

Figs. 4-5 presents the system design for 2.4 and $5 \mathrm{GHz}$, both figures illustrate allocated channels for each node. The channel allocation is done through greedy approach to avoid interference of conflict nodes of Fig. 3 while the greedy algorithm is applied in two steps for every node. $1^{\text {st }}$ channels were assigned up to $2^{\text {nd }}$ hop for the inter-flow links thereafter assign channel to direct connected hop for intra-flow link. All channels must be orthogonal and with minimum of one channel space. The processes will repeat for all nodes.

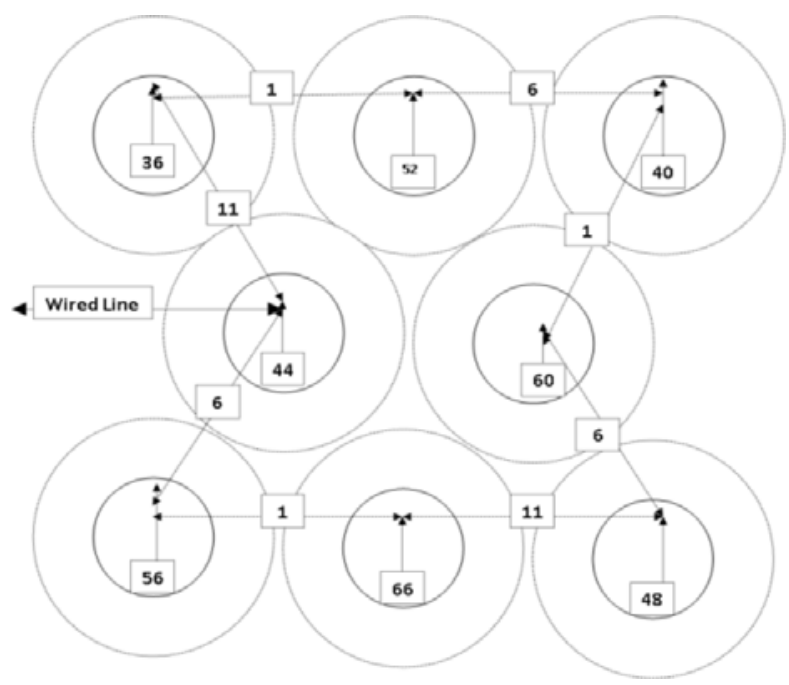

FIG. 4. SYSTEM DESIGN OF 2.4 GHZ BAND

Mehran University Research Journal of Engineering \& Technology, Volume 35, No. 4, October, 2016 [p-ISSN: 0254-7821, e-ISSN: 2413-7219] 
The design phase includes three scenarios named as No Orthogonal, Orthogonal_2.4GHz and Orthogonal_5GHz. For all scenarios term orthogonality refers to the consideration of orthogonality and channel spacing.

Scenarios involve 8 mesh nodes with three interfaces. Proposed strategy is associated with two interfaces reserved for backhaul while third interface uses omnidirectional antenna under $5 \mathrm{GHz}$ band and is reserved for user access. All subscribers are clients of FTP server with uniformly distributed traffic in this system.

First scenario shows the backhaul of $2.4 \mathrm{GHz}$ where random channel allocation is done without caring of orthogonality and channel space. In this scenario we have assign the static channels to backhaul links.

Second and third scenario shows the channel assignment considering proposed idea with the attention of orthogonality and channel space. Second scenario highlights the significance of proposed idea when backhaul is configured on $2.4 \mathrm{GHz}$. In the view of Fig. 5 it is cleared that channels assigned up to $1^{\text {st }}$ and $2^{\text {nd }}$ hop of every node is orthogonal and with proper channel space, while third scenario is based on channel assignment of

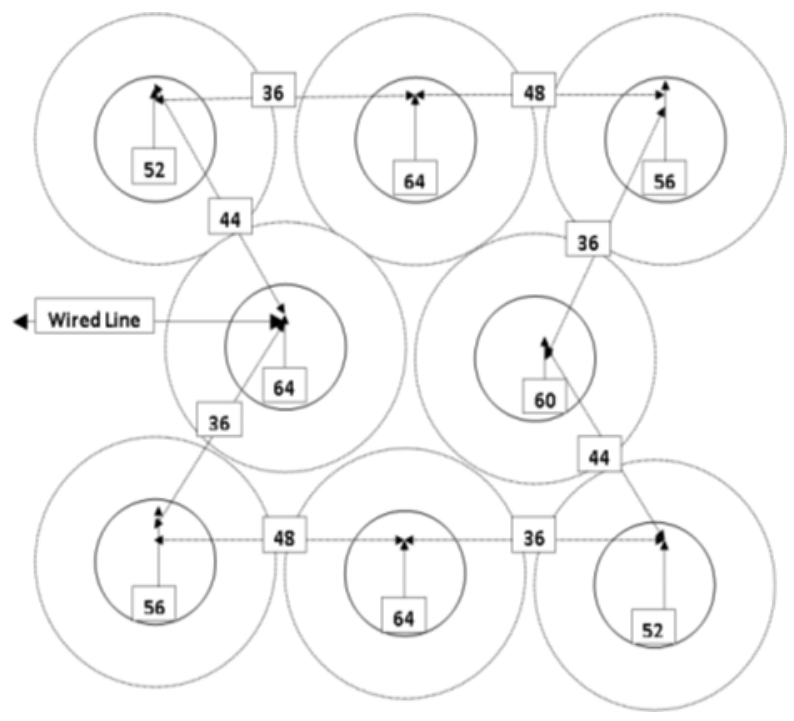

FIG. 5. SYSTEM DESIGN OF 5 GHZ BAND static channels of $5 \mathrm{GHz}$ band for backhauls links as shown in Fig. 5. 5GHz band have multiple orthogonal channels so there is the main consideration of handling channel spacing. The access points for all three scenarios are set of following default parameters; physical characteristic is set direct sequence, short retry limit sets on 7 , long retry limit sets on 4, transmitted power is 0,005 Watt, received power threshold -95, max. Life time is 0.5 seconds and access point beacon interval is 0.02 seconds.

\section{RESULTS AND DISCUSSION}

This section presents our simulation results which are evaluated from the three scenarios as discussed in our design section.

\section{$5.1 \quad$ Throughput}

Fig. 6 shows the throughput comparison of FTP server for three scenarios, the result shows that the FTP throughput of $5 \mathrm{GHz}$ higher than other two; the variation of throughput is almost lies in between 3200-3800 Kbits/ sec, where the performance is quite constant. While variation of 2.4GHz almost lies in between 2200-3200 Kbits/ sec, this shows the variation limits of $2.4 \mathrm{GHz}$ under proposed idea is double than the variation limit of $5 \mathrm{GHz}$ band.

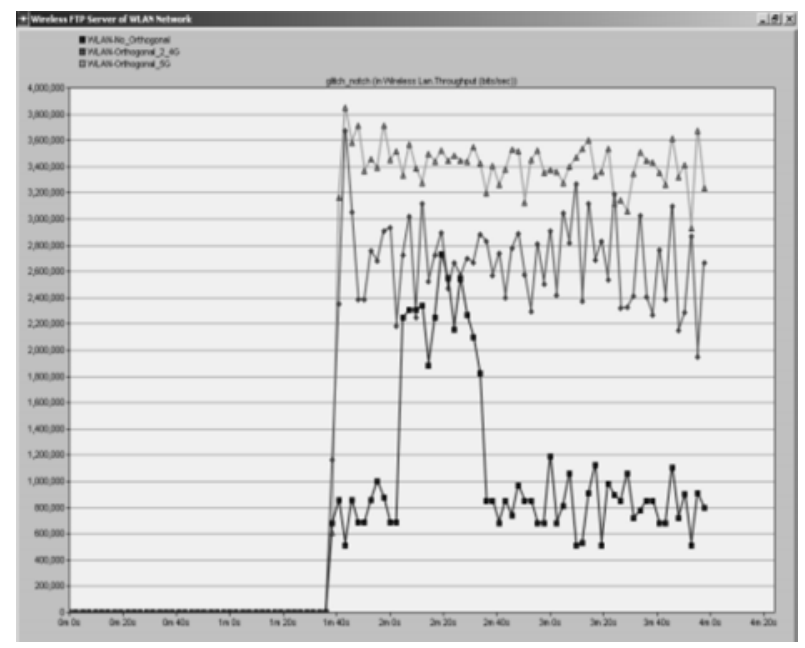

FIG. 6. FTP THROUGHPUT 
At the starting point $2.4 \mathrm{GHz}$ band touches to the level of $5 \mathrm{GHz}$ band then it goes down. This is because $5 \mathrm{GHz}$ band has all orthogonal channels there is only need of spaced channels (in our approach it is spaced by one channel), while $2.4 \mathrm{GHz}$ band has only 3 orthogonal channels. In Fig. 6 the lowest performance is of $2.4 \mathrm{GHz}$ non orthogonal scenario as the system faces channel interference which degraded its throughput.

Figs. 7-8 following the same performance pattern, Fig. 7 shows the LAN throughput and Fig. 8 displays the average throughput.

For the clear vision in Fig. 9 the throughput comparison of all scenarios are plotted through bar chart. Fig. 9 presents that for the $2.4 \mathrm{GHz}$ band the orthogonal channel arrangement enhance the throughput up to four times as compared to non-orthogonal channel arrangement, while under the suggested approach the throughput of $5 \mathrm{GHz}$ band is almost double than $2.4 \mathrm{GHz}$ band.

The previous studies employ different approaches to enhance the system throughput, their studies are mostly dealing with orthogonality in the reference of CCI while

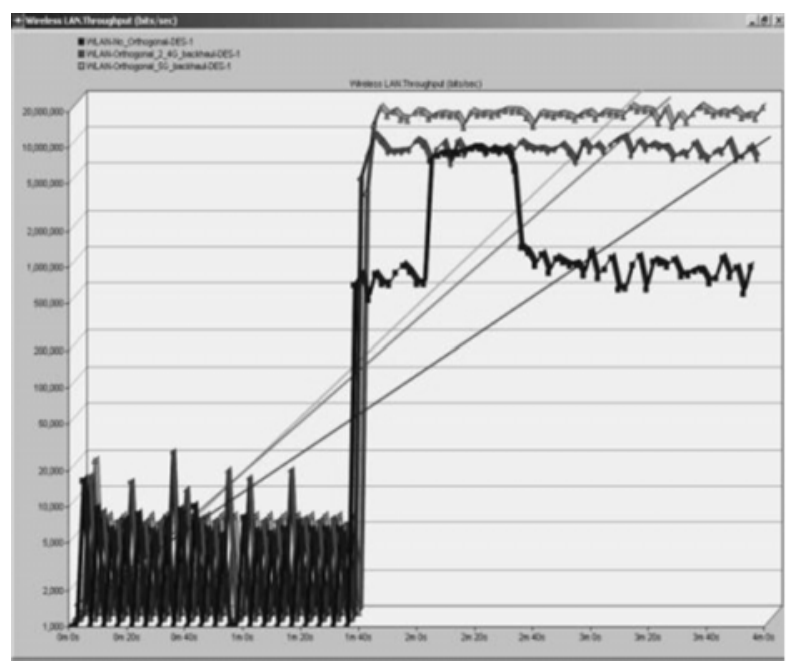

FIG. 7. LAN THROUGHPUT proposed effort is dealing with CCI and ACI, Thus reducing the effect of interference at its minimum level and enhance system throughput.

\subsection{Media Access Delay}

Fig. 10 shows the media access delay. These results show one major point to notice, that media access delay of 5 $\mathrm{GHz}$ band is more than $2.4 \mathrm{GHz}$ band for orthogonal channel assignment, it is because $5 \mathrm{GHz}$ band is more sensitive for long distance communication such as backhaul connectivity.

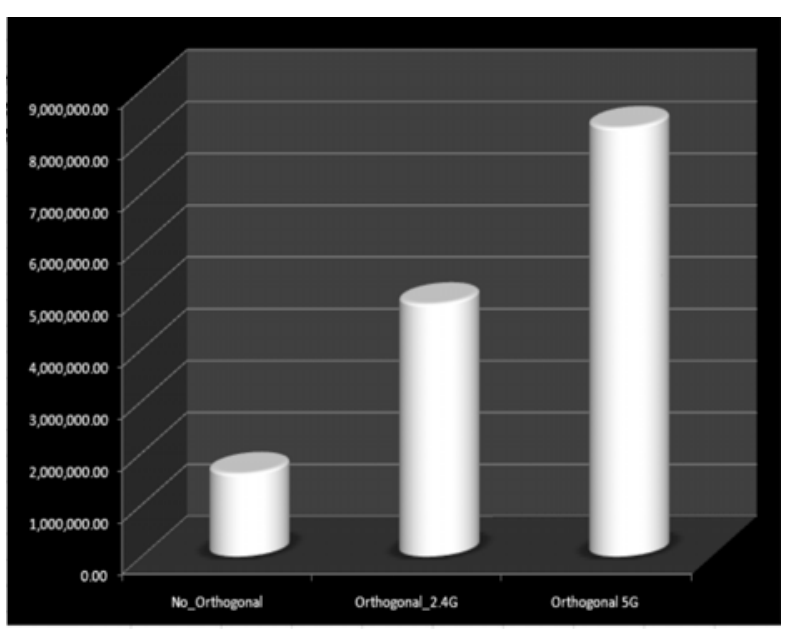

FIG. 9. AVERAGE THROUGHPUT

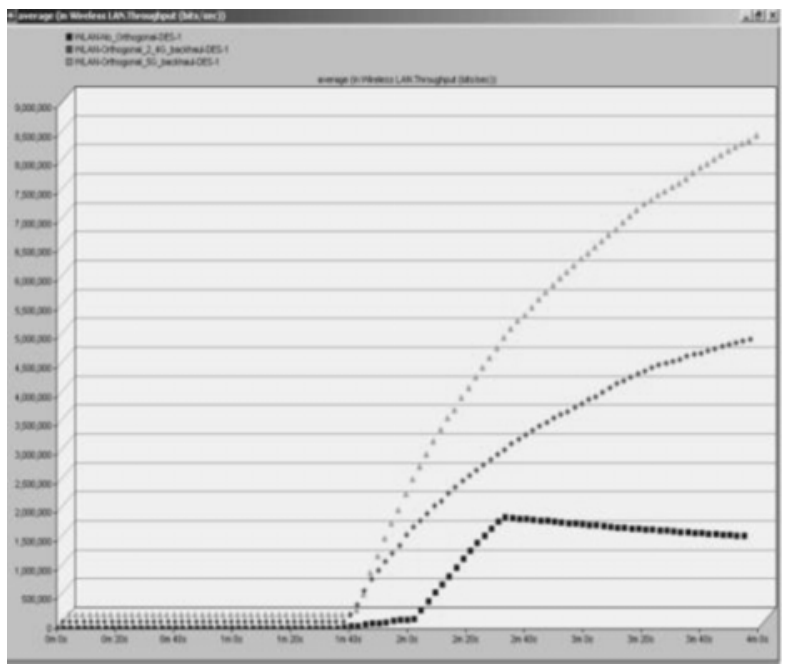

FIG. 8. AVERAGE THROUGHPUT

Mehran University Research Journal of Engineering \& Technology, Volume 35, No. 4, October, 2016 [p-ISSN: 0254-7821, e-ISSN: 2413-7219] 


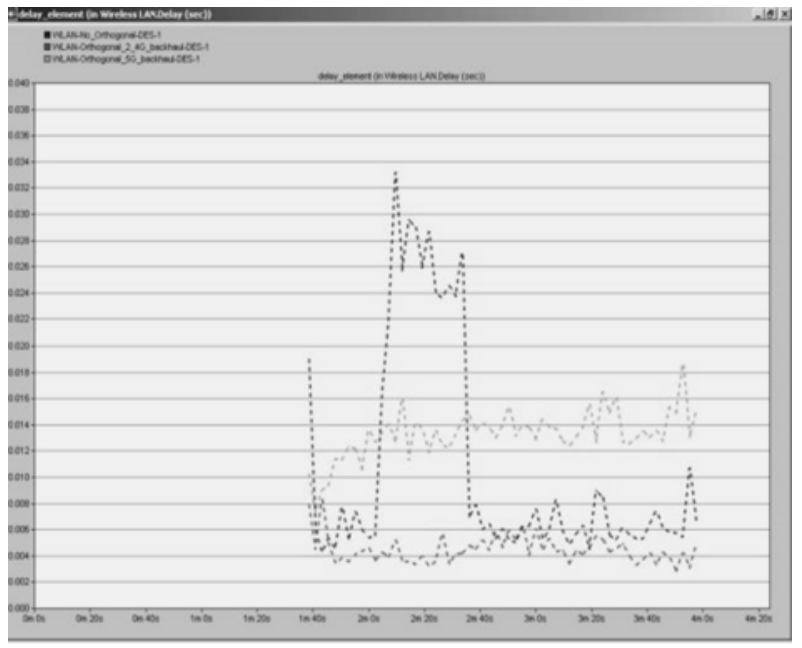

FIG. 10. MEDIA ACCESS DELAY

\section{CONCLUSION}

In this paper we have proposed a new channel scheme to address the interference in mesh networks. This work minimized the CCI and ACI with the proper selection of channels for nodes. Selection considers orthogonal and spaced channels for links. Through simulation work we achieved that our proposed channel assignment enhances the system performance in relation of network throughput and delay for both 2.4 and $5 \mathrm{GHz}$ bands. In future the proposed approach can be implemented for MIMO networks

\section{ACKNOWLEDGEMENTS}

Authors are thankful to Prof. Dr. Bhawani Shankar Chowdhry, Dean, Faculty of Electrical, Electronic \& Computer Engineering, Mehran University of Engineering \& Technology, Jamshoro, Pakistan, and Dr. Khalil-ur-Rehman Khoumbati, Department of Health Informatics, College of Public Health \& Health Informatics, Qassim University, Bukairyah, Saudi Arabia, for their guidelines that greatly improved the manuscript.

\section{REFERENCES}

[1] Rob, F., Krag, T., Fonda, C., Forster, J., Aichelle, C., Howard, I., and Zennaro, M., "Wireless Networking in the Developing World”, A Practical Guide to Planning and Building Low Cost Telecommunication Infrastructure, $2^{\text {nd }}$ Edition, Book Spring, pp. 12-100, London, January, 2007.

Malhotra, R., Gupta, V., and Bansal, R.K., "Simulation and Performance Analysis of Wired and Wireless Computer Networks”, International Journal of Computer Applications, Volume 14, No. 7, pp.11-17, 2011.

Jain, K., Padhye, J., Padmanabhan, V.N., and Qiu, L., "Impact of Interference on Multi-Hop Wireless Network Performance”, Wireless Networks, Volume 11, No. 4, pp. 471-487, 2005.

[4] Incel, O.D., VanHoesel, L., Jansen, P., and Havinga, P., "MC-LMAC: A Multi-Channel MAC Protocol for Wireless Sensor Networks”, Ad Hoc Networks, Elsevier, Volume 9, No. 1, pp. 73-94, 2001.

Raman, B., “Channel Allocation in 802.11-Based Mesh Networks”, Proceedings of 25th International Conference on Computer Communications, Volume 6, pp. 1-10, Barcelona, Spain, April, 2006.

[6] Chieochan, S., and Hossain, E., "Channel Assignment for Throughput Optimization in Multichannel Multi Radio Wireless Mesh Networks using Network Coding”, IEEE Transactions on Mobile Computing, Volume 12, No. 1, pp. 118-135, [ISSN: 1536-1233], January, 2013.

[7] Bukkapatanam, V., Franklin, A.A., and Murthy, C.S.RV., Bukkapatanam, A.F., and Murthy, C., "Using Partially Overlapped Channels for End-to-End Flow Allocation and Channel Assignment in Wireless Mesh Networks", IEEE International Conference on Communication, pp. 1-6, Dresden, Germany, June, 2009.

[8] Hoque, M.A., Hong, X., and Afroz, F., "Multiple Radio Channel Assignment Utilizing Partially Overlapped Channels”, Proceedings of IEEE Conference on Global Telecommunication, pp. 1-7, Honolulu, HI, November, 2009. 
[9] Zhou, K., Jia, X., Chang, Y., and Tang, X., "Partially Overlapping Channel Assignment for WLANs Using SINR Interference Model”, International Journal of Communication Systems, Volume 27, No. 11, pp. 3082-3095, 2014.

[10] Duarte, P.B., Fadlullah, Z.M., Hashimoto, K., and Kato, N., "Partially Overlapped Channel Assignment on Wireless Mesh Network Backbone”, Proceedings of IEEE Conference on Global Communication, pp. 1-5, Miami, FL, December, 2010.

Duarte, P.B., Fadlullah, Z.M., Vasilakos, A.V., and Kato, N., "On the Partially Overlapped Channel Assignment on Wireless Mesh Network Backbone: A Game Theoretic Approach”, IEEE Journal Selected Areas in Communications, Volume 30, No. 1, pp. 119-127, 2012.

Bejerano, Y., Han, S.J., and Kumar, A., "Efficient LoadBalancing Routing for Wireless Mesh Networks”, Computer Networks, Volume 51, No. 10, pp. 2450-2466, 2007.
[13] Avallone, S., and Akyildiz, I.F., "A Channel Assignment Algorithm for Multi-Radio Wireless Mesh Networks”, Computer Communications, Volume 31, No. 7, pp. 1343-1353, 2008.

[14] Tsao, S.L., Su, J.J., Huang, K.L., Shih, Y.C., and Tseng, C.C., "An End to End Channel Allocation Scheme for a Wireless Mesh Network", International Journal of Communication Systems, Volume 27, No. 12, pp. 4407-4429, 2014.

[15] Kim, T.S., Yang, Y., Hou, J.C., and Krishnamurthy, S.V., "Resource Allocation for QoS Support in Wireless Mesh Networks”, IEEE Transactions on Wireless Communications, Volume 12, No. 5, pp. 2046-2054, 2013.

[16] Ananth, C.A., Selvakumar, K., and Manikannan, K., "End-to-End Flow Allocation and Channel Assignment in MC-MR Wireless Mesh Networks”, International Journal of Computer Networks \& Wireless Communications, [ISSN: 2250-3501], Volume 3, No. 6, pp. 466-470, December, 2013. 